JOURNAL OF THE

AMERICAN MATHEMATICAL SOCIETY

Volume 22, Number 2, April 2009, Pages 309-329

S 0894-0347(08)00624-3

Article electronically published on October 17, 2008

\title{
ASYMPTOTIC ENUMERATION AND LIMIT LAWS OF PLANAR GRAPHS
}

\author{
OMER GIMÉNEZ AND MARC NOY
}

\section{Introduction AND Statement of RESUlts}

A graph is planar if it can be embedded in the plane, or in the sphere, so that no two edges cross at an interior point. A planar graph together with a particular embedding is called a map. There is a rich theory of counting maps, started by Tutte in the 1960's. However, in this paper we are interested in counting graphs as combinatorial objects, regardless of how many nonequivalent topological embeddings they may have. As we are going to see, this makes the counting considerably more difficult.

In this paper we obtain a precise asymptotic estimate for the number of labelled planar graphs on $n$ vertices, and we establish limit laws for several parameters in random labelled planar graphs. In particular, we show that the number of edges in random planar graphs is asymptotically normal and that the number of connected components in a random planar graph is distributed asymptotically as a shifted Poisson law. Additional Gaussian and Poisson limit laws for random planar graphs are derived.

From now on, all graphs are labelled, finite and simple. Let $g_{n}$ be the number of planar graphs on $n$ vertices. A superadditivity argument [12] shows that the following limit exists:

$$
\gamma=\lim _{n \rightarrow \infty}\left(g_{n} / n !\right)^{1 / n} .
$$

Until recently, the constant $\gamma$ was known only within certain bounds, namely

$$
26.18<\gamma<30.06
$$

The lower bound results from the work of Bender, Gao and Wormald [1]. They show that, if $b_{n}$ is the number of 2-connected planar graphs, then

$$
\lim _{n \rightarrow \infty}\left(b_{n} / n !\right)^{1 / n} \approx 26.18 .
$$

Hence $\gamma$ is at least this value.

The upper bound is based on the fact that an unlabelled planar graph on $n$ vertices can be encoded with at most $\alpha n$ bits for some constant $\alpha$. If this is the case, then $g_{n} \leq 2^{\alpha n} n$ !, and so $\gamma \leq 2^{\alpha}$. The first such result was obtained by Turán [16] with the value $\alpha=12$. This has been improved over the years and presently the

Received by the editors August 3, 2005.

2000 Mathematics Subject Classification. Primary 05A16, 05C30; Secondary 05C10.

Key words and phrases. Planar graph, random planar graph, asymptotic enumeration, limit law, normal law, analytic combinatorics.

The first author's research was supported in part by Project MTM2005-08618C02-01.

(C)2008 American Mathematical Society Reverts to public domain 28 years from publication 
best result is $\alpha \approx 4.91$, obtained by Bonichon et al. 3 . Since $2^{4.91} \approx 30.06$, the upper bound follows.

Recently the present authors [10] were able to obtain, using numerical methods, the approximation $\gamma \approx 27.2268$. In this paper we determine $\gamma$ exactly as an analytic expression. Moreover, we find a precise asymptotic estimate for the number of planar graphs.

In order to state our results, we need to define the following functions of the complex variable $t$ :

$$
\begin{aligned}
\xi= & \frac{(1+3 t)(1-t)^{3}}{16 t^{3}} \\
Y= & \frac{(1+2 t)}{(1+3 t)(1-t)} \exp \left(-\frac{t^{2}(1-t)\left(18+36 t+5 t^{2}\right)}{2(3+t)(1+2 t)(1+3 t)^{2}}\right)-1, \\
r= & \frac{1}{16} \sqrt{1+3 t}(1-t)^{3} t^{-3} \exp (A), \\
A= & \frac{\log (1+t)(3 t-1)(1+t)^{3}}{16 t^{3}}+\frac{\log (1+2 t)(1+3 t)(1-t)^{3}}{32 t^{3}} \\
& +\frac{(1-t)\left(185 t^{4}+698 t^{3}-217 t^{2}-160 t+6\right)}{64 t(1+3 t)^{2}(3+t)}
\end{aligned}
$$

According to Lemma 2 in [1, $Y$ is a one-to-one function from $(0,1)$ onto $(0, \infty)$. Let $v(y)$ be the inverse function of $Y(t)$. Let $t_{0}=v(1)$, that is, the unique value such that $Y\left(t_{0}\right)=1$. The approximate value is $t_{0} \approx 0.62637$.

Theorem 1. Let $g_{n}$ be the number of planar graphs on $n$ vertices. Then

$$
g_{n} \sim g \cdot n^{-7 / 2} \gamma^{n} n !
$$

where $\gamma=r\left(t_{0}\right)^{-1}$. The approximate values are $\gamma=27.22688$ and $g \approx 0.42609$. $10^{-5}$.

The exact expression for the multiplicative constant $g$ is too long to be stated here in detail. It will be shown in the proof of Theorem 1 that $g=e^{C_{0}} c$, where $C_{0}=\xi+B_{0}+B_{2}, c=B_{0}\left(1-2 B_{4} / \xi\right)^{-5 / 2}$, and all functions are evaluated at $t_{0}$. The definition of the functions $B_{i}$ can be found in the appendix.

As we show later, for the number $c_{n}$ of connected planar graphs on $n$ vertices, we have the estimate

$$
c_{n} \sim c \cdot n^{-7 / 2} \gamma^{n} n !
$$

where $\gamma$ and $c \approx 0.41043 \cdot 10^{-5}$ are as before.

The proof of Theorem 1 is based on singularity analysis of generating functions; see [5, 6]. Let $g_{n}, c_{n}$ and $b_{n}$ be as before. As we show in the next section, there are two equations linking the exponential generating functions

$$
B(x)=\sum b_{n} x^{n} / n !, \quad C(x)=\sum c_{n} x^{n} / n !, \quad G(x)=\sum g_{n} x^{n} / n ! .
$$

The dominant singularity of $B(x)$ was determined in [1]; we are able to obtain the dominant singularities of $C(x)$ and $G(x)$, which are both equal to $\rho=\gamma^{-1}$.

In Section 2 we review the preliminaries needed for the proof. In Section 3 we find an explicit expression for the generating function $B(x, y)$ of 2-connected planar graphs counted according to the number of vertices and edges. This is a key technical result in the paper, which allows us to obtain a full bivariate singular expansion of $B(x, y)$. The explicit expression obtained for the function $\beta(x, y, z, w)$ 
in the statement of Lemma 5 suggests that we are in fact integrating a rational function. This is indeed the case as we explain later.

In Section 4 we determine expansions of $C(x)$ and $G(x)$ of square-root type at the dominant singularity $\rho$, and then we apply "transfer theorems" [5, 6] to obtain estimates for $c_{n}$ and $g_{n}$.

The singular expansions of $C(x)$ and $G(x)$ can be extended to the corresponding bivariate generating functions $C(x, y)$ and $G(x, y)$ near $y=1$. This allows us to prove in Section 5, using perturbation of singularities [6], a normal limit law for the number of edges in random planar graphs. To our knowledge, this problem was first posed in [4].

Throughout this paper, we say that a sequence of random variables $X_{n}$ with mean $\mu_{n}$ and variance $\sigma_{n}^{2}$ has a normal limit law if the normalized variables $X_{n}^{*}=\left(X_{n}-\right.$ $\left.\mu_{n}\right) / \sigma_{n}$ converge in law to the standard normal distribution $\mathcal{N}(0,1)$; convergence in law means, as usual, pointwise convergence of the corresponding distribution functions.

Theorem 2. Let $X_{n}$ denote the number of edges in a random planar graph with $n$ vertices. Then $X_{n}$ is asymptotically normal and the mean $\mu_{n}$ and variance $\sigma_{n}^{2}$ satisfy

$$
\mu_{n} \sim \kappa n, \quad \sigma_{n}^{2} \sim \lambda n
$$

where

$$
\kappa=-\frac{\rho^{\prime}(1)}{\rho(1)}, \quad \lambda=-\frac{\rho^{\prime \prime}(1)}{\rho(1)}-\frac{\rho^{\prime}(1)}{\rho(1)}+\left(\frac{\rho^{\prime}(1)}{\rho(1)}\right)^{2},
$$

and $\rho(y)=r(v(y))$ (recall that $v$ is inverse to $Y$ ). The approximate values are $\kappa \approx 2.21326$ and $\lambda \approx 0.43034$.

The same is true, with the same constants, for connected random planar graphs.

As a consequence, since $\sigma_{n}=o\left(\mu_{n}\right)$, the number of edges is concentrated around its expected value; that is, for every $\epsilon>0$ we have

$$
\operatorname{Prob}\left\{\left|X_{n}-\kappa n\right|>\epsilon n\right\} \rightarrow 0, \quad \text { as } n \rightarrow \infty .
$$

Previously it had been proved that $\operatorname{Prob}\left\{X_{n}<\alpha n\right\} \rightarrow 0$ and $\operatorname{Prob}\left\{X_{n}>\beta n\right\} \rightarrow 0$, as $n \rightarrow \infty$, for some constants $\alpha$ and $\beta$. The best values achieved so far were $\alpha \approx 1.85$ (shown in [8, improving upon [4]) and $\beta \approx 2.44$ (shown in [3, improving upon [14). Theorem 2 shows that in fact there is only one constant that matters, namely $\kappa$.

The previous theorem shows convergence in distribution to the normal law. However, in this setting it is often the case that one can also prove a local limit law, that is, convergence to the density function of the normal law. We prove such a local limit law and we derive large deviation estimates for the number of edges in random planar graphs. In the next statement, as later in the paper, $g_{n, q}$ and $c_{n, q}$ denote, respectively, the number of planar graphs and connected planar graphs with $n$ vertices and $q$ edges; $\rho(y), G_{5}(y)$ and $C_{5}(y)$ are computable analytic functions to be introduced later.

Theorem 3. Let $\mu$ be a fixed ratio in the open interval $(1,3)$. Take $u>0$ such that $-u \rho^{\prime}(u) / \rho(u)=\mu$. Then, as $n$ goes to $\infty$,

$$
g_{n,\lfloor\mu n\rfloor} \sim n ! G_{5}(u) \frac{\rho(u)^{-n} u^{-\lfloor\mu n\rfloor}}{\sqrt{2 \pi n} \Gamma(-5 / 2) \sigma n^{7 / 2}},
$$




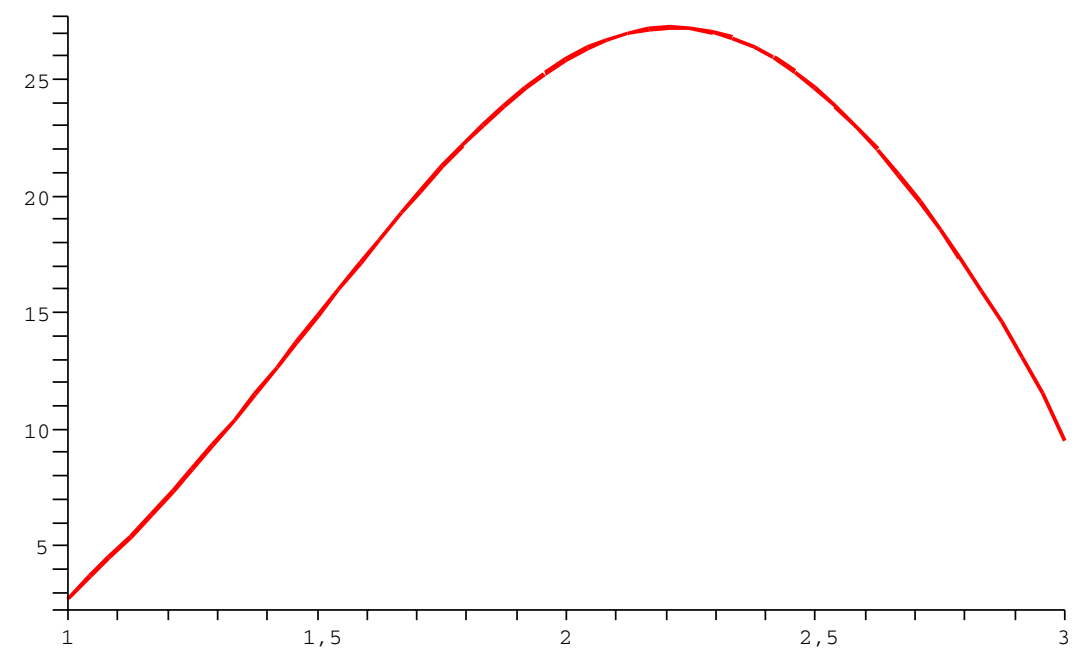

FiguRE 1. The growth ratio of planar graphs with $n$ vertices and $\lfloor\mu n\rfloor$ edges

where

$$
\sigma^{2}=-u^{2} \frac{\rho^{\prime \prime}(u)}{\rho(u)}-u \frac{\rho^{\prime}(u)}{\rho(u)}+u^{2} \frac{\rho^{\prime}(u)^{2}}{\rho(u)^{2}} .
$$

The same is true for the number of connected planar graphs $c_{n,\lfloor\mu n\rfloor}$ if we replace $G_{5}(u)$ by $C_{5}(u)$ in (1.3).

The previous result makes more precise a recent result from [9], where the authors show that

$$
\lim _{n \rightarrow \infty} \frac{1}{n} \log \frac{g_{n,\lfloor\mu n\rfloor}}{n !}=\lambda(\mu),
$$

where $\lambda(\mu)$ is a continuous function of $\mu$. A direct consequence of Theorem 3 is that

$$
\lambda(\mu)=-\mu \log (u)-\log (\rho(u))
$$

where $u$ depends on $\mu$ as in the statement of the theorem. Notice that $\lambda(\mu)$ is an analytic function of $\mu$. Figure 1 shows the plot of $\exp (\lambda(\mu))$, that is, the growth ratio of planar graphs with $n$ vertices and $\lfloor\mu n\rfloor$ edges. The limit of $\exp (\lambda(\mu))$ as $\mu \rightarrow 1$ is equal to $e$, which is the growth ratio of labelled trees; the limit as $\mu \rightarrow 3$ is equal to $256 / 27$, which is the growth ratio of triangulations [17. (Tutte's result is for unlabelled triangulations, but a triangulation has at most a linear number of automorphisms.)

Next we turn our attention to the following problem, considered in 12. Let $H$ be a graph on the vertex set $\{1, \ldots, h\}$, and let $G$ be a graph on the vertex set $\{1, \ldots, n\}$, where $n>h$. Let $W \subset V(G)$ with $|W|=h$, and let $r_{W}$ denote the least element in $W$. Following [12, we say that $H$ appears at $W$ in $G$ if (a) the increasing bijection from $\{1, \ldots, h\}$ to $W$ gives an isomorphism between $H$ and the induced subgraph $G[W]$ of $G$ and (b) there is exactly one edge in $G$ between $W$ and the rest of $G$, and this edge is incident with the root $r_{W}$. 
Let $a_{H}(G)$ be the number of appearances of $H$ in $G$, that is, the number of sets $W \subset V(G)$ such that $H$ appears at $W$ in $G$. Let $\alpha$ be $\left(9 e^{2}(h+2)\right)^{-1} \rho^{h} / h$ !. It is shown in [12] that if $G_{n}$ is a random planar graph on $n$ vertices, then

$$
\operatorname{Pr}\left\{a_{H}\left(G_{n}\right) \leq \alpha n\right\}<e^{-\alpha n},
$$

for $n$ large enough. The next result describes more precisely the asymptotic behavior of the number of appearances of $H$ in random planar graphs.

Theorem 4. Let $H$ be a fixed rooted connected planar graph with h vertices. Let $X_{n}$ denote the number of appearances of $H$ in a random planar graph with $n$ vertices. Then $X_{n}$ is asymptotically normal and the mean $\mu_{n}$ and variance $\sigma_{n}^{2}$ satisfy

$$
\mu_{n} \sim \frac{\rho^{h}}{h !} n, \quad \sigma_{n}^{2} \sim \rho n,
$$

where $\rho=\gamma^{-1}$ and $\gamma$ is as in Theorem 1. Moreover, for every $\alpha<\rho^{n} / n$ ! and every $\beta>\rho^{n} / n$ ! we have for $n$ large enough

$$
\operatorname{Pr}\left\{X_{n}<\alpha n\right\}<\left(\frac{u^{\alpha}}{x(u) \rho}\right)^{n}, \quad \operatorname{Pr}\left\{X_{n}>\beta n\right\}<\left(\frac{u^{\beta}}{x(u) \rho}\right)^{n},
$$

where $x(u)$ is the solution of

$$
x e^{(u-1) x^{k} / k !}=\rho
$$

and $u$ is related to $Z$, where $Z$ is either $\alpha$ or $\beta$, by the equation

$$
-u \frac{x^{\prime}(u)}{x(u)}=Z .
$$

Another parameter, the number of 2-connected components in a random connected planar graph, also follows a normal distribution.

Theorem 5. Let $X_{n}$ denote the number of blocks (2-connected components) in a random connected planar graph with $n$ vertices. Then $X_{n}$ is asymptotically normal and the mean $\mu_{n}$ and variance $\sigma_{n}^{2}$ satisfy

$$
\mu_{n} \sim \zeta n, \quad \sigma_{n}^{2} \sim \zeta n,
$$

where $\zeta=\log (R / \rho) \approx 0.039052$.

Next we turn to a different parameter, the number of connected components in random planar graphs.

Theorem 6. Let $X_{n}$ denote the number of connected components in a random planar graph with $n$ vertices. Then $X_{n}-1$ is distributed asymptotically as a Poisson law of parameter $\nu$, where $\nu=C(\rho) \approx 0.037439$.

The above result is an improvement upon what was known so far. It is shown in [12] that $Y_{n}$ is stochastically dominated by $1+Y$, where $Y$ is a Poisson law $P(1)$; Theorem 6 shows that in fact $Y_{n}$ is asymptotically $1+P(\nu)$. The following direct corollary to Theorem 6 is worth mentioning.

Corollary 1. (i) The probability that a random planar graph is connected is asymptotically equal to $e^{-\nu} \approx 0.96325$. (ii) The expected number of components in a random planar graph is asymptotically equal to $1+\nu \approx 1.03744$. 
Our last result is the following. Let $\mathcal{A}$ be a family of connected planar graphs, and let $A(x)=\sum A_{n} x^{n} / n$ ! be the corresponding generating function. Assume that the radius of convergence of $A(x)$ is strictly larger than $\rho=\gamma^{-1}$, the radius of convergence of $C(x)$; this is equivalent to saying that $\mathcal{A}$ is exponentially smaller than the family $\mathcal{C}$ of all connected planar graphs.

Theorem 7. Assume $\mathcal{A}$ is a family of connected planar graphs that satisfies the previous condition, and let $X_{n}$ denote the number of connected components that belong to $\mathcal{A}$ in a random planar graph with $n$ vertices. Then $X_{n}$ is distributed asymptotically as a Poisson law of parameter $A(\rho)$.

If we take $\mathcal{A}$ as the family of graphs isomorphic to a fixed connected planar graph $H$ with $n$ vertices, then

$$
A(x)=\frac{n !}{|\operatorname{Aut}(H)|} \cdot \frac{x^{n}}{n !}=\frac{x^{n}}{|\operatorname{Aut}(H)|},
$$

where $\operatorname{Aut}(H)$ is the group of automorphisms of $H$. In particular, if $H$ is a single vertex, we obtain that the number of isolated vertices in a random planar graph tends to a Poisson law $P(\rho)=P\left(\gamma^{-1}\right)$. This proves a conjecture by McDiarmid, Steger and Welsh 12 .

As a different application of Theorem 7 we have the following. Recall that $B(x)$ is the generating function of 2-connected planar graphs.

Corollary 2. Let $X_{n}$ denote the number of connected components which are 2connected in a random planar graph with $n$ vertices. Then $X_{n}$ tend to a Poisson law of parameter $B(\rho) \approx 0.00068370$.

We wish to emphasize that the approach that eventually has led to the enumeration of planar graphs has a long history. Whitney's theorem 21] guarantees that a 3-connected graph has a unique embedding in the sphere; hence the problem of counting 3-connected graphs is in essence equivalent to counting 3-connected maps (planar graphs with a specific embedding). This last problem was solved by Mullin and Schellenberg 13 using the approach developed by Tutte in his seminal papers on counting maps (see, for instance, [18]). The next piece is due to Tutte [19]: a 2 -connected graph decomposes uniquely into 3-connected "components". Tutte's decomposition implies equations connecting the generating functions of 3-connected and 2-connected planar graphs, which were obtained by Walsh 20, using the results of Trakhtenbrot [15. This in turn was used by Bender, Gao and Wormald [1] to solve the problem of counting 2-connected planar graphs; their work is most relevant to us and is in fact the starting point of our research. Finally, the decomposition of connected graphs into 2-connected components and the decomposition of arbitrary graphs into connected components imply equations connecting the corresponding generating functions. Analytic methods, together with a certain amount of algebraic manipulation, become then the main ingredients in our solution.

\section{Preliminaries}

In this section and in the rest of the paper we use the language and basic results of Analytic Combinatorics, as in the forthcoming book of Flajolet and Sedgewick [6]. For the sake of completeness, we state the main results we use in this paper (Corollary VI.1, Theorems IX.10 and IX.13 in [6]). 
Proposition 1 (Transfer Theorem; simplified version). Assume that $f(z)$ is analytic in a domain $\Delta=\Delta(\phi, R)$, where $R>1,0<\phi<\pi / 2$ and

$$
\Delta(\phi, R)=\{z: z \neq 1,|z|<R,|\operatorname{Arg}(z-1)|>\phi\} .
$$

If, as $z \rightarrow 1$ in $\Delta$,

$$
f(z) \sim(1-z)^{-\alpha}
$$

then

$$
\left[z^{n}\right] f(z) \sim \frac{n^{\alpha-1}}{\Gamma(\alpha)}
$$

Proposition 2 (Quasi-Powers Theorem; algebraic singularities). Let $f(z, u)$ be a bivariate function that is bivariate analytic at $(0,0)$ with nonnegative coefficients there. Assume that $f$ admits in $\mathcal{D}=\{|z| \leq r\} \times\{|u-1|<\epsilon\}$, for some $r>0$ and $\epsilon>0$, the representation

$$
f(z, u)=A(z, u)+B(z, u) C(z, u)^{-\alpha},
$$

where $A, B$ and $C$ are analytic in $\mathcal{D}$ such that $C(z, 1)=0$ has a unique simple root $\rho<r$ in $|z| \leq r$ and $B(\rho, 1) \neq 0$. Moreover, neither $\partial_{z} C(\rho, 1)$ nor $\partial_{u} C(\rho, 1)$ are 0 , so there exists a nonconstant $\rho(u)$ analytic at $u=1$ such that $C(\rho(u), u)=0$ and $\rho=\rho(1)$. Finally, $\rho(u)$ is such that

$$
-\frac{\rho^{\prime \prime}(1)}{\rho(1)}-\frac{\rho^{\prime}(1)}{\rho(1)}+\left(\frac{\rho^{\prime}(1)}{\rho(1)}\right)^{2}
$$

is nonzero. Then the random variable with probability generating function

$$
p_{n}(u)=\frac{\left[z^{n}\right] f(z, u)}{\left[z^{n}\right] f(z, 1)}
$$

converges in distribution to a Gaussian variable. The mean $\mu_{n}$ and the standard deviation $\sigma_{n}$ converge asymptotically to $\mu$ and $\sigma \sqrt{n}$, where $\mu$ is $-\rho^{\prime}(1) / \rho(1)$ and $\sigma^{2}$ is given by (2.1).

Proposition 3 (Local Limit Law; simplified version). Let $f(x, u)$ satisfy the conditions of Proposition 2, If $\rho(u)$ attains uniquely its minimum on the circle $|u|=1$ at $u=1$, then the sequence $p_{n,\lfloor\mu n\rfloor}$ is asymptotically $(\sqrt{2 \pi n} \sigma)^{-1}$.

Now we discuss the generating functions that appear in this paper. Recall that $g_{n}, c_{n}$ and $b_{n}$ denote, respectively, the number of planar graphs, connected planar graphs, and 2-connected planar graphs on $n$ vertices. The corresponding exponential generating functions are related as follows.

Lemma 1. The series $G(x), C(x)$ and $B(x)$ satisfy the following equations:

$$
G(x)=\exp (C(x)), \quad x C^{\prime}(x)=x \exp \left(B^{\prime}\left(x C^{\prime}(x)\right)\right),
$$

where $C^{\prime}(x)$ and $B^{\prime}(x)$ are derivatives with respect to $x$.

Proof. The first equation is standard, given the fact that a planar graph is a set of connected planar graphs, and the set construction in labelled structures corresponds to taking the exponential of the corresponding exponential generating function.

The second equation follows from a standard argument on the decomposition of a connected graph into 2-connected components. Take a connected graph rooted at a vertex $v$, hence the generating function $x C^{\prime}(x)$. Now $v$ belongs to a set of 2-connected components (including single edges), each of them rooted at vertex $v$, 
hence the term $\exp \left(B^{\prime}\right)$. Finally, in each of the 2-connected components, replace every vertex by a rooted connected graph; this explains the substitution $B^{\prime}\left(x C^{\prime}(x)\right)$. Details can be found, for instance, in [11, p. 10].

Let $b_{n, q}$ be the number of 2-connected planar graphs with $n$ vertices and $q$ edges, and let

$$
B(x, y)=\sum b_{n, q} y^{q} \frac{x^{n}}{n !}
$$

be the corresponding bivariate generating function. Notice that $B(x, 1)=B(x)$. The generating functions $C(x, y)$ and $G(x, y)$ are defined analogously. Since the parameter "number of edges" is additive under taking connected and 2-connected components, the previous lemma can be extended as follows.

Lemma 2. The series $G(x, y), C(x, y)$ and $B(x, y)$ satisfy the following equations:

$$
G(x, y)=\exp (C(x, y)), \quad x \frac{\partial}{\partial x} C(x, y)=x \exp \left(\frac{\partial}{\partial x} B\left(x \frac{\partial}{\partial x} C(x, y), y\right)\right) .
$$

In the remainder of the section we recall the necessary results from [1]. Define the series $M(x, z)$ by means of the expression

$$
M(x, z)=x^{2} z^{2}\left(\frac{1}{1+x z}+\frac{1}{1+z}-1-\frac{(1+U)^{2}(1+V)^{2}}{(1+U+V)^{3}}\right),
$$

where $U(x, z)$ and $V(x, z)$ are algebraic functions given by

$$
U=x z(1+V)^{2}, \quad V=z(1+U)^{2} .
$$

In the next result and in the rest of the paper, all logarithms are natural.

Lemma 3 (Bender et al. [1]). We have

$$
\frac{\partial B(x, y)}{\partial y}=\frac{x^{2}}{2}\left(\frac{1+D(x, y)}{1+y}\right)
$$

where $D=D(x, y)$ is defined implicitly by $D(x, 0)=0$ and

$$
\frac{M(x, D)}{2 x^{2} D}-\log \left(\frac{1+D}{1+y}\right)+\frac{x D^{2}}{1+x D}=0 .
$$

Moreover, the coefficients of $D(x, y)$ are nonnegative.

There is a small modification in equation (2.4) with respect to [1. We must consider the graph consisting of a single edge as being 2-connected; otherwise Lemmas 1 and 2 would not hold. Hence the term of lowest degree in the series $B(x, y)$ is $y x^{2} / 2$.

Let us comment on the previous equations. The algebraic generating function $M$ corresponds to (rooted) 3-connected planar maps. The decomposition of a 2-connected graph into 3-connected components implies equations (2.4) and (2.5). The generating function $D(x, y)$ is that of planar networks, which are special graphs with two distinguished vertices. 
We define the following functions of the complex variable $t$. The appendix contains additional functions that are introduced later.

$$
\begin{aligned}
\xi & =\frac{(1+3 t)(1-t)^{3}}{16 t^{3}} \\
Y & =\frac{(1+2 t)}{(1+3 t)(1-t)} \exp \left(-\frac{t^{2}(1-t)\left(18+36 t+5 t^{2}\right)}{2(3+t)(1+2 t)(1+3 t)^{2}}\right)-1, \\
\alpha & =144+592 t+664 t^{2}+135 t^{3}+6 t^{4}-5 t^{5}, \\
\beta & =3 t(1+t)\left(400+1808 t+2527 t^{2}+1155 t^{3}+237 t^{4}+17 t^{5}\right), \\
D_{0} & =\frac{3 t^{2}}{(1-t)(1+3 t)}, \\
D_{2} & =-\frac{48 t^{2}(1+t)(1+2 t)^{2}\left(18+6 t+t^{2}\right)}{(1+3 t) \beta} \\
D_{3} & =384 t^{3}(1+t)^{2}(1+2 t)^{2}(3+t)^{2} \alpha^{3 / 2} \beta^{-5 / 2} .
\end{aligned}
$$

Let us notice a slight change in terminology: functions $\xi$ and $Y$ are denoted, respectively, $x_{0}$ and $y_{0}$ in 1]; also, we correct a typo, namely a $t$ factor that was missing in the expression for $D_{2}$.

A key fact is that for $y$ in a suitable small neighborhood of 1 , the equation $Y(t)=y$ has a unique solution in $t=t(y)$. Then define

$$
R(y)=\xi(t(y)) .
$$

In the next lemma, $D_{i}(y)$ stands for $D_{i}(t(y))$. This applies too to functions $B_{i}(y)$ and $C_{i}(y)$ that we introduce later in the paper.

Lemma 4 (Bender et al. 11). For fixed $y$ in a small neighborhood of $1, R(y)$ is the unique dominant singularity of $D(x, y)$. Moreover, $D(x, y)$ has a branch-point at $R(y)$, and the singular expansion at $R(y)$ is of the form

$$
D(x, y)=D_{0}(y)+D_{2}(y) X^{2}+D_{3}(y) X^{3}+\mathcal{O}\left(X^{4}\right),
$$

where $X=\sqrt{1-x / R(y)}$ and the $D_{i}(y)$ are as before.

The previous lemma is the key result used in [1] to prove the estimate

$$
b_{n} \sim b \cdot n^{-7 / 2} R^{-n} n !
$$

where $b$ is a constant and $R=R(1) \approx 0.038191$.

\section{Analysis of $B(x, y)$}

From equation (2.4), it follows that

$$
B(x, y)=\frac{x^{2}}{2} \int_{0}^{y} \frac{1+D(x, t)}{1+t} d t .
$$

Our goal is to obtain an expression for $B(x, y)$ as a function of $x, y$ and $D(x, y)$ that, although more complex, does not contain an integral. Recall that the algebraic function $U$ is defined in (2.3) and $D$ is defined in Lemma 3

Lemma 5. Let $W(x, z)=z(1+U(x, z))$. The generating function $B(x, y)$ of 2connected planar graphs admits the following expression as a formal power series:

$$
B(x, y)=\beta(x, y, D(x, y), W(x, D(x, y))),
$$


where

and

$$
\beta(x, y, z, w)=\frac{x^{2}}{2} \beta_{1}(x, y, z)-\frac{x}{4} \beta_{2}(x, z, w),
$$

$$
\begin{aligned}
\beta_{1}(x, y, z) & =\frac{z(6 x-2+x z)}{4 x}+(1+z) \log \left(\frac{1+y}{1+z}\right)-\frac{\log (1+z)}{2}+\frac{\log (1+x z)}{2 x^{2}}, \\
\beta_{2}(x, z, w) & =\frac{2(1+x)(1+w)\left(z+w^{2}\right)+3(w-z)}{2(1+w)^{2}}-\frac{1}{2 x} \log \left(1+x z+x w+x w^{2}\right) \\
& +\frac{1-4 x}{2 x} \log (1+w)+\frac{1-4 x+2 x^{2}}{4 x} \log \left(\frac{1-x+x z-x w+x w^{2}}{(1-x)\left(z+w^{2}+1+w\right)}\right) .
\end{aligned}
$$

Proof. From equation (3.1) we obtain

$$
B(x, y)=\frac{x^{2}}{2} \log (1+y)+\frac{x^{2}}{2} \int_{0}^{y} \frac{D(x, t)}{1+t} d t .
$$

We integrate by parts and obtain

$$
\int_{0}^{y} \frac{D(x, t)}{1+t} d t=\log (1+y) D(x, y)-\int_{0}^{y} \log (1+t) \frac{\partial D(x, t)}{\partial t} d t .
$$

From now on $x$ is a fixed value. Now notice that from (2.5) it follows that

$$
\phi(u)=-1+(1+u) \exp \left(-\frac{M(x, u)}{2 x^{2} u}-\frac{x u^{2}}{1+x u}\right),
$$

is an inverse of $D(x, y)$, in the sense that $\phi(D(x, y))=y$. In the last integral we change variables $s=D(x, t)$, so that $t=\phi(s)$. Then

$$
\begin{aligned}
\int_{0}^{y} \log (1+t) \frac{\partial D(x, t)}{\partial t} d t & =\int_{0}^{D(x, y)}\left(\log (1+s)-\frac{x s^{2}}{1+x s}\right) d s \\
& -\int_{0}^{D(x, y)} \frac{M(x, s)}{2 x^{2} s} d s .
\end{aligned}
$$

The first integral has a simple primitive and we are left with an integral involving $M(x, z)$. Summing up, we have

$$
B(x, y)=\Theta(x, y, D(x, y))+\frac{1}{4} \int_{0}^{D(x, y)} \frac{M(x, s)}{s} d s,
$$

where $\Theta$ is the elementary function

$$
\Theta(x, y, z)=\frac{x^{2}}{2}\left(z+\frac{1}{2} z^{2}+(1+z) \log \frac{1+y}{1+z}\right)-\frac{x}{2} z+\frac{1}{2} \log (1+x z) .
$$

Now we concentrate on the last integral. From (2.2) and (2.3) it follows that

$$
\int_{0}^{D} \frac{M(x, s)}{s} d s=-x \int_{0}^{D} \frac{(1+U)^{2} U}{(1+U+V)^{3}} d s+x^{2} \int_{0}^{D} s\left(\frac{1}{1+x s}+\frac{1}{1+s}-1\right) d s,
$$

where $U$ and $V$ are considered as functions of $x$ and $s$ and where for simplicity we write $D=D(x, y)$ from now on.

The second integral is elementary. For the first one, notice that from the definition $W(x, s)=s(1+U(x, s))$, we obtain

$$
\frac{(1+U)^{2} U}{(1+U+V)^{3}}=\frac{W-s}{W(1+W)^{3}} .
$$


Since $W$ satisfies the equation

$$
x s^{2}+\left(1+2 x W^{2}\right) s+W\left(x W^{3}-1\right)=0,
$$

the functional inverse of $W(x, s)$ with respect to the second variable is equal to

$$
-t^{2}-\frac{1-\sqrt{1+4 x t+4 x t^{2}}}{2 x}
$$

where we use $t$ to denote the new variable.

It follows that

$$
\int_{0}^{D} \frac{W-s}{W(1+W) 3} d s=\int_{0}^{W(x, D)} \frac{\left(Q-1-2 x t-2 x t^{2}\right)(2 Q t-2 t-1)}{2 x t(1+t)^{3} Q} d t,
$$

where for simplicity we write

$$
Q(x, t)=\sqrt{1+4 x t+4 x t^{2}} .
$$

The last integral can be solved explicitly with the help of a computer algebra system such as MAPLE and we obtain as a primitive the function

$$
\begin{gathered}
\frac{1-2(t+4 x+4 x t)}{4 x(1+t)^{2}}-\frac{1+2 x(1+t)}{2 x(1+t)^{2}} Q^{3}+\left(2+4 x t+\frac{1+2(t-x-t x)}{4 x(1+t)^{2}}\right) Q \\
+\frac{2 x^{2}-4 x+1}{4 x} \log \left(\frac{Q+(1-2 x-2 x t)}{Q-(1-2 x-2 x t)}\right)-\frac{1}{2 x} \log (Q+1+2 x t)+\frac{1-4 x}{2 x} \log (1+t) .
\end{gathered}
$$

Finally we have to replace $t$ with $W(x, D)$ in the previous equation. The expression (3.4) and equation (3.5) imply that

$$
Q(x, W(x, D))=1+2 x\left(D+W(x, D)^{2}\right) .
$$

Hence when replacing $t$ with $W(x, D)$, we obtain an expression in $x, D$ and $W(x, D)$ that is free of square roots. A routine computation, combined with the intermediate equation (3.3), gives the final expression for $B(x, y)$ as claimed.

The function $\beta$ in the previous lemma looks like the primitive of a rational function. This can be explained as follows (we are grateful to P. Flajolet for this observation). The algebraic equation satisfied by $U$ (here $x$ is considered as a parameter) is

$$
u-x y\left(1+y(1+u)^{2}\right)^{2}=0 .
$$

It can be checked (for instance, using the MAPLE package algcurves) that this equation in $u$ and $y$ defines a rational curve, that is, a curve of genus zero, and so it admits a rational parametrization $(u(t), y(t))$. Now an integral $\int R(s, U(x, s)) d s$, where $R$ is a rational function, becomes the integral of a rational function after the change of variables $s=y(t)$. In particular, this applies to the integral in equation (3.3).

The former lemma can be used to obtain the singular expansion of $B(x, y)$. The function $R(y)$ is defined in (2.6) and $B_{0}, B_{2}, B_{4}, B_{5}$ are analytic functions of $y$ given in the appendix. Again $B_{i}(y)$ stands for $B_{i}(t)$, where $t$ is the unique solution of $Y(t)=y$ in a neighborhood of 1 .

Lemma 6. For fixed $y$ in a small neighborhood of 1 , the dominant singularity of $B(x, y)$ is equal to $R(y)$. The singular expansion at $R(y)$ is of the form

$$
B(x, y)=B_{0}(y)+B_{2}(y) X^{2}+B_{4}(y) X^{4}+B_{5}(y) X^{5}+\mathcal{O}\left(X^{6}\right),
$$

where $X=\sqrt{1-x / R(y)}$ and the $B_{i}$ are analytic functions in a neighborhood of 1 . 
Proof. Consider the expression for $B(x, y)$ in Lemma 5 as a function of $x, y$ and $D(x, y)$. A simple analysis shows that, for $y$ close to 1 , the only singularities come from the singularities of $D(x, y)$; hence the first claim follows.

For the second assertion, substitute the singular expansions of $D(x, y)$ and $U(x, D(x, y)$ ) (taken, respectively, from Lemma 4 and the appendix) for $D(x, y)$ and $U(x, D(x, y))$ in (3.2) (recall that $W=z(1+U))$. Next set $x=\xi(t)\left(1-X^{2}\right)$ and $y=Y(t)$ as functions of $t$, and expand the resulting expression. That the coefficients $B_{i}$ are as claimed in the appendix is a tedious but routine computation that we performed with the help of MAPLE. In particular, the coefficients of $X$ and $X^{3}$ vanish identically in $y$ (or in $t$ ). The $B_{i}$ are analytic since they are elementary functions of the $D_{i}$.

\section{Asymptotic estimates}

In order to prove Theorem 1, first we need to locate the dominant singularity $\rho=\gamma^{-1}$ of $G(x)$. Since $G(x)=\exp (C(x))$, the functions $G(x)$ and $C(x)$ have the same singularities; hence from now on we concentrate on $C(x)$.

We rewrite the second equation in Lemma 1 as

$$
F(x)=x \exp \left(B^{\prime}(F(x))\right),
$$

where $F(x)=x C^{\prime}(x)$. Notice that the singularities of $B^{\prime}(x)$ and $F(x)$ are the same, respectively, as those of $B(x)$ and $C(x)$. From (4.1) it follows that

$$
\psi(u)=u e^{-B^{\prime}(u)}
$$

is the functional inverse of $F(x)$. The dominant singularity of $\psi$ is the same as that of $B(x)$, which according to Lemma 6 is equal to $R=R(1)$. In order to determine the dominant singularity $\rho$ of $F(x)$, we have to decide which of the following possibilities hold; see Proposition IV.4 in [6] for an explanation.

(1) There exists $\tau \in(0, R)$ (necessarily unique) such that $\psi^{\prime}(\tau)=0$. Then $\psi$ ceases to be invertible at $\tau$ and $\rho=\psi(\tau)$.

(2) We have $\psi^{\prime}(u) \neq 0$ for all $u \in(0, R)$. Then $\rho=\psi(R)$.

The condition $\psi^{\prime}(\tau)=0$ is equivalent to $B^{\prime \prime}(\tau)=1 / \tau$. Since $B^{\prime \prime}(u)$ is increasing (the series $B(u)$ has positive coefficients) and $1 / u$ is decreasing, we are in case (2) if and only if $B^{\prime \prime}(R)<1 / R$. Next we show that this is the case.

Claim 1. Let $R$ be, as before, the radius of convergence of $B(x)$. Then $B^{\prime \prime}(R)<$ $1 / R$.

Proof. Lemma 6 implies that $B^{\prime \prime}(R)=2 B_{4} / R^{2}$ (see (4.3) below). Hence the inequality becomes $2 B_{4}<R$. It holds because $R \approx 0.0381$ and $B_{4} \approx 0.000767$.

Let us remark that in a related problem, counting series-parallel graphs, a very similar situation appears but the analogous $\psi$ function does have a maximum in its domain of definition [2].

We are now ready for the main result.

Proof of Theorem 1. As we have seen in Claim 1, the dominant singularity of $F(x)$ is at $\rho=\psi(R)$. In order to obtain the singular expansion of $F(x)$ at $\rho$, we have to invert the singular expansion of $\psi(u)$ at $R$. 
The expansion of $B^{\prime}(x)$ follows directly by differentiating the one in Lemma 6.

$$
B^{\prime}(x)=-\frac{1}{R}\left(B_{2}+2 B_{4} X^{2}+\frac{5}{2} B_{5} X^{3}\right)+\mathcal{O}\left(X^{4}\right) .
$$

Because of $\psi(x)=x \exp \left(-B^{\prime}(x)\right)$, by functional composition we obtain

$$
\psi(x)=R e^{B_{2} / R}\left(1+\left(\frac{2 B_{4}}{R}-1\right) X^{2}+\frac{5 B_{5}}{2 R} X^{3}\right)+\mathcal{O}\left(X^{4}\right) .
$$

Since we are inverting at the singularity, $F(x)$ also has a singular expansion of square-root type

$$
F(x)=F_{0}+F_{1} X+F_{2} X^{2}+F_{3} X^{3}+\mathcal{O}\left(X^{4}\right),
$$

with the difference that now $X=\sqrt{1-x / \rho}$. Given that $F(x)$ and $\psi(x)$ are functional inverses, the $F_{i}$ can be found by indeterminate coefficients, and they turn out to be, in terms of $R$ and the $B_{i}$,

$$
F_{0}=R, \quad F_{1}=0, \quad F_{2}=\frac{R^{2}}{2 B_{4}-R}, \quad F_{3}=-\frac{5}{2} B_{5}\left(1-2 B_{4} / R\right)^{-5 / 2} .
$$

The singular expansion of $C(x)$ is obtained by integrating $C^{\prime}(x)=F(x) / x$, and one gets

$$
C(x)=C_{0}+C_{2} X^{2}+C_{4} X^{4}+C_{5} X^{5}+\mathcal{O}\left(X^{6}\right) .
$$

The $C_{i}$, except $C_{0}$, are computed easily in terms of the $F_{i}$ in equation (4.4), and they turn out to be

$$
C_{2}=-F_{0}, \quad C_{4}=-\frac{F_{0}+F_{2}}{2}, \quad C_{5}=-\frac{2}{5} F_{3} .
$$

By singularity analysis (Proposition [1), we obtain the estimate

$$
c_{n} \sim c \cdot n^{-7 / 2} \rho^{-n} n !,
$$

where $c=C_{5} / \Gamma(-5 / 2)$.

However, the coefficient $C_{0}=C(\rho)$ is indeterminate after the integration of $F(x) / x$ and is needed later. To compute it, we start by integrating by parts

$$
C(x)=\int_{0}^{x} \frac{F(s)}{s} d s=F(x) \log x-\int_{0}^{x} F^{\prime}(s) \log s d s .
$$

We change variables $t=F(s)$, so that $s=\psi(t)=t e^{-B^{\prime}(t)}$, and the last integral becomes

$$
\int_{0}^{F(x)} \log \psi(t) d t=\int_{0}^{F(x)}\left(\log t-B^{\prime}(t)\right) d t=F(x) \log F(x)-F(x)-B(F(x)) .
$$

Hence

$$
C(x)=F(x) \log x-F(x) \log F(x)+F(x)+B(F(x)) .
$$

Taking into account that $F(\rho)=R$ and $B(R)=B_{0}$, we get

$$
C_{0}=C(\rho)=R \log \rho-R \log R+R+B_{0} .
$$

A simple computation shows that, equivalently,

$$
C_{0}=R+B_{0}+B_{2} \text {. }
$$


The final step is simpler since $G(x)=e^{C(x)}$. We apply the exponential function to (4.5) and obtain the singular expansion

$$
G(x)=e^{C_{0}}\left(1+C_{2} X^{2}+\left(C_{4}+\frac{1}{2} C_{2}^{2}\right) X^{4}+C_{5} X^{5}\right)+\mathcal{O}\left(X^{6}\right),
$$

where again $X=\sqrt{1-x / \rho}$. Again by singularity analysis, we obtain the estimate

$$
g_{n} \sim g \cdot n^{-7 / 2} \rho^{-n} n !,
$$

where $g=e^{C_{0}} c$. Finally, since $\rho=\psi(R)=R e^{-B^{\prime}(R)}$ and $B^{\prime}(R)=-B_{2} / R$, we get

$$
\rho=R e^{B_{2} / R}, \quad \gamma=\rho^{-1}=\frac{1}{R} e^{-B_{2} / R} .
$$

The constants $c, g$ and $\rho$ can be found using the known value of $R$ and the expressions for the $B_{i}$ in the appendix; the approximate values in the statement have been computed using these expressions.

Notice that the probability that a random planar graph is connected is equal to $c_{n} / g_{n} \sim c / g=e^{-C_{0}}$. This result reappears later in Theorem 6.

\section{Gaussian limit LaWs}

The proofs in this section are based on bivariate singular expansions and perturbation of singularities. To simplify the notation, in this section we denote by $f^{\prime}(x, y)$ the derivative of a bivariate function with respect to $x$.

Proof of Theorem 2, We rewrite the second equation in Lemma 2 as

$$
F(x, y)=x \exp \left(B^{\prime}(F(x, y), y)\right),
$$

where $F(x, y)=x C^{\prime}(x, y)$. It follows that, for $y$ fixed,

$$
\psi(u, y)=u e^{-B^{\prime}(u, y)}
$$

is the functional inverse of $F(x, y)$.

We know from the previous section that $\psi^{\prime}(u, y)$ does not vanish for $y=1$ and $u \in(0, R)$ and that $\rho=\psi(R)$ is the dominant singularity of $F(x)$. Hence by continuity the same is true for $y$ close to 1 , and the dominant singularity of $F(x, y)$ is at

$$
\rho(y)=\psi(R(y), y)=R(y) e^{-B^{\prime}(R(y), y)} .
$$

Given the analytic expressions for the functions involved, the univariate singular expansion of $\psi(x)$ extends to an expansion of $\psi(x, y)$ for $y$ fixed. The same is true then for $F(x, y)$ and $C(x, y)$, and we obtain a bivariate expansion

$$
C(x, y)=C_{0}(y)+C_{2}(y) X^{2}+C_{4}(y) X^{4}+C_{5}(y) X^{5}+\mathcal{O}\left(X^{6}\right)
$$

where the $C_{i}(y)$ are analytic functions, and now $X=\sqrt{1-x / \rho(y)}$.

Then Proposition 2 implies a limit normal law for the number of edges in random connected planar graphs, with expectation and variance linear in $n$. The constants $\kappa$ and $\lambda$ in the statement of Theorem 2 are given by

$$
\kappa=-\frac{\rho^{\prime}(1)}{\rho(1)}, \quad \lambda=-\frac{\rho^{\prime \prime}(1)}{\rho(1)}-\frac{\rho^{\prime}(1)}{\rho(1)}+\left(\frac{\rho^{\prime}(1)}{\rho(1)}\right)^{2},
$$

where $\rho^{\prime}(y)=d \rho(y) / d y$. Since $G(x, y)$ and $C(x, y)$ have the same dominant singularities $\rho(y)$, the previous statement also holds for arbitrary planar graphs, with the same values of $\kappa$ and $\lambda$. 
In order to determine the parameters exactly, we need only an explicit expression for $\rho(y)$. The expansion (4.3) extends to an expansion of $B^{\prime}(x, y)$, whose constant term is $B^{\prime}(R(y), y)=-B_{2}(y) / R(y)$. Hence from (5.3) it follows that

$$
\rho(y)=R(y) \exp \left(B_{2}(y) / R(y)\right) .
$$

The appendix contains an explicit expression for $\rho(y)=q(t)$ as a function of $t$. The necessary derivatives are computed as $\rho^{\prime}(y)=q^{\prime}(t) / Y^{\prime}(t)$, and the same goes for $\rho^{\prime \prime}(y)$. The approximate values in the statement have been computed in this way.

Proof of Theorem 3. Consider the generating function $C_{u}(x, y)=C(x, u y)$, where $u$ is a fixed constant. In this situation the singularity $\rho_{u}(y)$ of $C_{u}$ is given by $\rho(u y)$, and the associated probabilities $p_{n, k}^{u}$ of $C_{u}$ are

$$
p_{n, k}^{u}=\frac{\left[y^{k}\right]\left[x^{n}\right] C_{u}(x, y)}{\left[x^{n}\right] C_{u}(x, 1)}=\frac{u^{k} c_{n, k}}{n !\left[x^{n}\right] C(x, u)} .
$$

In order to apply Proposition 3 to $C_{u}$, we need to know the singularities of $C(x, y)$ when $y$ is away from 1. The following claim extends Claim 1 and shows that the bivariate singularity expansions given in the proof of Theorem 2 hold for every $y$.

Claim 2. Let $R(y)$ be the radius of convergence of $B(x, y)$ for $y$ fixed. Then $B^{\prime \prime}(R(y), y)<1 / R(y)$.

Proof. As in the proof of Claim 1, it is enough to show that $2 B_{4}(y)<R(y)$ for $y \in(0, \infty)$, equivalently, that $2 B_{4}(t)<\xi(t)$ for $t \in(0,1)$. We bound the logarithm that appears in the expression for $B_{4}$ (see the appendix) as

$$
\log \left(\frac{1+t}{\sqrt{1+2 t}}\right) \leq \frac{1+t}{\sqrt{1+2 t}}-1
$$

Let $\widetilde{B}_{4}$ be the function obtained by substituting the logarithm in $B_{4}$ for the righthand side in the previous inequality. Then it is enough to show that

$$
2 \widetilde{B}_{4}(t)<\xi(t) \quad \text { for } t \in(0,1) .
$$

Since both $\widetilde{B}_{4}$ and $\xi$ are rational functions, the problem reduces to showing that a certain polynomial (in fact, of degree 20) with integer coefficients has no root in $(0,1)$. We have checked that this is indeed the case using MAPLE.

Another requirement is that $\rho(z)$ attains uniquely its minimum on $|z|=u$ at $z=u$. Suppose there exists $w \neq u$ with $|w|=u$ such that $|\rho(w)| \leq \rho(u)$. It follows from (5.3) that $R(z)$ is equal to $F(\rho(z), z)$, and since $F(x, y)$ has nonnegative coefficients, $|R(w)|=|F(\rho(w), w)| \leq F(\rho(u), u)=R(u)$. However, this contradicts the fact that $R(z)$ attains uniquely its minimum on $|z|=u$ at $z=u$, as shown in [1, Lemma 3].

Now Proposition 3 applied to $C_{u}$ yields

$$
p_{n,\lfloor\mu(u) n\rfloor}^{u} \sim \frac{1}{\sqrt{2 \pi n} \sigma(u)},
$$


where $\mu(u)$ and $\sigma(u)$ are given by

$$
\begin{gathered}
\mu(u)=-\frac{\rho_{u}^{\prime}(1)}{\rho_{u}(1)}=-\frac{u \rho^{\prime}(u)}{\rho(u)} \\
\sigma(u)^{2}=-\frac{\rho_{u}^{\prime \prime}(1)}{\rho_{u}(1)}-\frac{\rho_{u}^{\prime}(1)}{\rho_{u}(1)}+\left(\frac{\rho_{u}^{\prime}(1)}{\rho_{u}(1)}\right)^{2}=-u^{2} \frac{\rho^{\prime \prime}(u)}{\rho(u)}-u \frac{\rho^{\prime}(u)}{\rho(u)}+\left(u \frac{\rho^{\prime}(u)}{\rho(u)}\right)^{2} .
\end{gathered}
$$

Theorem 3 follows by combining equations (5.4) and (5.5) for $k=\lfloor\mu(u) n\rfloor$ and using the asymptotic expression of $\left[x^{n}\right] C(x, y)$ for $y=u$. The value $\mu$ is constrained to the interval $(1,3)$ since $\lim _{u \rightarrow 0} \mu(u)=1$ and $\lim _{u \rightarrow \infty} \mu(u)=3$.

Proof of Theorem 4, Let us recall equation (4.1), i.e.,

$$
F(x)=x \exp \left(B^{\prime}(F(x))\right),
$$

where $F(x)=x C^{\prime}(x)$ is the generating function of rooted connected planar graphs. In order to mark appearances of $H$, we have to look at the root $r$ of a rooted connected graph $G$ and the blocks to which it belongs; recall this is encoded in the term $\exp \left(B^{\prime}(F(x))\right)$. We are interested in the blocks which are equal to a single edge $r v$ and within these blocks to the situation where vertex $v$ is replaced by a copy of $H$. In this case we mark an appearance of $H$ with the secondary variable $y$. If we let $f(x, y)$ be the corresponding generating function, then the previous discussion translates into the equation

$$
f(x, y)=x \exp \left(B^{\prime}(f(x, y))+(y-1) \frac{x^{h}}{h !}\right) .
$$

Notice that from the definition of appearances we do not need to take into account the automorphisms of $H$; if a copy of $H$ is substituted for vertex $v$, there is only one way to do it once the labels are selected, hence the term $x^{h} / h$ !.

In fact, $f(x, y)$ is not the exact counting series, since it does not take into account the possibility that the root $r$ belongs to a copy of $H$ that appears in $G$. This can be accounted for as follows. The generating function of rooted connected graphs where the root belongs to an appearance of $H$ is $f(x, y) x^{h} /(h-1)$ !, since the root can appear in any of the $h$ vertices of $H$. Hence the generating function that counts exactly all appearances is

$$
g(x, y)=f(x, y)+(y-1) \frac{x^{h}}{(h-1) !} f(x, y) .
$$

Since $f(x, y)$ and $g(x, y)$ have the same dominant singularity for any fixed $y$, it does not matter which one we choose for singularity analysis; hence in the rest of the proof we work with $f(x, y)$, defined through (5.6).

Equation (5.6) can be rewritten as

$$
f(x, y)=\zeta(x, y) \exp \left(B^{\prime}(f(x, y))\right),
$$

where $\zeta(x, y)=x \exp \left((y-1) x^{h} / h\right.$ !). Comparing the previous equation with (4.1), it follows that

$$
f(x, y)=F(\zeta(x, y)) .
$$

Given that $\rho$ is the dominant singularity of $F(x)$, the dominant singularity of $f(x, y)$ for fixed $y$ is the smallest value $\tau(y)$ satisfying

$$
\zeta(\tau(y), y)=\tau(y) \exp \left((y-1) \frac{\tau(y)^{h}}{h !}\right)=\rho .
$$


Clearly $\tau(1)=\rho$. In order to compute $\tau^{\prime}(y)$, we differentiate (5.7), set $y=1$, and obtain $\tau^{\prime}(1)=-\rho^{h+1} / h$ !. To compute $\tau^{\prime \prime}(1)$, we differentiate again and, after a simple computation, we get

$$
-\frac{\tau^{\prime}(1)}{\tau(1)}=\frac{\rho^{h}}{h !}, \quad-\frac{\tau^{\prime \prime}(1)}{\tau(1)}-\frac{\tau^{\prime}(1)}{\tau(1)}+\left(\frac{\tau^{\prime}(1)}{\tau(1)}\right)^{2}=\rho .
$$

From the singular expansion of $F(x)$ at $\rho$, we derive a corresponding bivariate singular expansion of $f(x, y)$ at $\tau(y)$, and again a normal limit law follows from Proposition 2. As in the previous proof, a large deviation estimate also follows, and from this we obtain the bounds in (1.5); the details are omitted to avoid repetition.

Proof of Theorem 5. The proof is similar to the previous proofs, and so we omit some details. The generating function $C_{1}(x, y)$ of connected planar graphs according to the number of vertices and blocks satisfies the equation

$$
x C_{1}^{\prime}(x, y)=x \exp \left(y B^{\prime}\left(x C_{1}^{\prime}(x, y)\right)\right),
$$

where $B(x)$ is the univariate generating function of 2-connected planar graphs.

Let $F_{1}(x, y)=x C_{1}^{\prime}(x, y)$. Then, for $y$ fixed,

$$
\psi_{1}(u, y)=u e^{-y B^{\prime}(u)}
$$

is the functional inverse of $F_{1}(x, y)$. The dominant singularity of $\psi_{1}(u, y)$ is at $R$, which in this case is independent of $y$, and the dominant singularity of $F_{1}(x, y)$ is at

$$
\rho_{1}(y)=\psi_{1}(R, y)=R e^{-y B^{\prime}(R)} .
$$

Again we have bivariate singular expansions whose coefficients are analytic functions of $y$, and the quasi-powers theorem implies asymptotic normality of the parameter. The asymptotic expressions for the expected value and variance are obtained as before, but in this case the computations are particularly easy, since

$$
\rho_{1}^{\prime}(y)=-\rho_{1}(y) B^{\prime}(R) .
$$

We know that $\rho=\psi(R)=R e^{-B^{\prime}(R)}$; hence

$$
\zeta=-\frac{\rho_{1}^{\prime}(1)}{\rho_{1}(1)}=B^{\prime}(R)=\log (R / \rho) \approx 0.039052 .
$$

A similar computation gives

$$
-\frac{\rho_{1}^{\prime \prime}(1)}{\rho_{1}(1)}-\frac{\rho_{1}^{\prime}(1)}{\rho_{1}(1)}+\left(\frac{\rho_{1}^{\prime}(1)}{\rho_{1}(1)}\right)^{2}=B^{\prime}(R)=\zeta .
$$

\section{Poisson Limit LaWs}

As opposed to the proofs in the previous section, to prove Theorems 6 and 7 . univariate asymptotics is enough.

Proof of Theorem 6. Let $\nu=C(\rho)=C_{0}$, the evaluation of $C(x)$ at its dominant singularity. For fixed $k$, the generating function of planar graphs with exactly $k$ connected components is

$$
\frac{1}{k !} C(x)^{k} \text {. }
$$

For fixed $k$, taking the $k$-th power of the singular expansion (4.5) of $C(x)$, we have

$$
\left[x^{n}\right] C(x)^{k} \sim k C_{0}^{k-1}\left[x^{n}\right] C(x) .
$$


Hence the probability that a random planar graphs has exactly $k$ components is asymptotically

$$
\frac{\left[x^{n}\right] C(x)^{k} / k !}{\left[x^{n}\right] G(x)} \sim \frac{k C_{0}^{k-1}}{k !} e^{-C_{0}}=\frac{\nu^{k-1}}{(k-1) !} e^{-\nu},
$$

as was to be proved.

Proof of Theorem 7. The proof is similar to the previous one. The generating function of planar graphs with no component belonging to $\mathcal{A}$ is $\exp (C(x)-A(x))$. Hence the generating function of planar graphs with exactly $k$ components in $\mathcal{A}$ is

$$
\frac{1}{k !} A(x)^{k} \exp (C(x)-A(x))=\frac{1}{k !} A(x)^{k} e^{-A(x)} G(x) .
$$

The same kind of simple calculation as before gives that the probability that a random planar graphs has exactly $k$ components in $\mathcal{A}$ is asymptotically

$$
\frac{A(\rho)^{k}}{k !} e^{-A(\rho)} \text {. }
$$

This finishes the proof of the theorem.

\section{Concluding Remarks}

We have found a solution to the problem of counting labelled planar graphs; however, counting unlabelled planar graphs appears to be much more difficult. If $u_{n}$ is the number of unlabelled planar graphs on $n$ vertices, then it is known that the limit

$$
\gamma_{u}=\lim _{n \rightarrow \infty}\left(u_{n}\right)^{1 / n}
$$

exists and that $\gamma<\gamma_{u}$, where $\gamma$ is as in Theorem 1 (see [12]). The reason for the strict inequality $\gamma<\gamma_{u}$ is that, contrary to what happens for unrestricted graphs, a planar graph has with high probability an exponential number of automorphisms [12].

The best upper bound obtained so far is $\gamma_{u}<30.06$. This is proved in [3] by showing that an unlabelled planar graph with $n$ vertices can be encoded with $4.91 n$ bits. On the other hand, our determination of $\gamma$ provides a lower bound on $\gamma_{u}$ and shows that at least $4.76 \approx \log _{2} \gamma$ bits per vertex are needed.

We believe that to determine $\gamma_{u}$ exactly is a very hard problem, not to speak of determining the subexponential behavior of $u_{n}$. The reason is that the equations connecting the generating functions of labelled planar graphs with different connectivity requirements do not hold anymore in the unlabelled case.

A related problem is to estimate the number of planar graphs with a given number of edges. In [3] it is proved that an unlabelled planar graph with $m$ edges can be encoded with $2.82 \mathrm{~m}$ bits. We can show that at least 2.59 bits per edge are needed, as follows.

The coefficient of $y^{m}$ in $G(1, y)$ is equal to

$$
h_{m}=\sum_{n} \frac{g_{n, m}}{n !},
$$

where $g_{n, m}$ is the number of labelled planar graphs with $n$ vertices and $m$ edges. Since a graph on $n$ vertices has at most $n$ ! automorphisms, the number of unlabelled planar graphs with $m$ edges is at least $h_{m}$. 
The exponential growth of the $h_{m}$ is determined by the smallest singularity $\tau$ of $G(1, y)$. Since the smallest singularity of $G(x, y)$ for fixed $y$ is $\rho(y)$, as given in (5.3), it follows that $\tau$ is the smallest solution to $\rho(\tau)=1$. It can be computed exactly with the expressions in the appendix and it turns out that

$$
\lim _{n \rightarrow \infty}\left(h_{m}\right)^{1 / m}=\tau \approx 6.03 \approx 2^{2.59} .
$$

Finally, let us mention that the explicit expressions we have obtained for the generating functions of labelled planar graphs have been applied to the design of very efficient algorithms for generating random planar graphs uniformly [7].

\section{APPENDIX}

Here we list the functions $B_{0}, B_{2}, B_{4}, B_{5}$ that have been used in the previous sections, as functions of $t$. As has been explained already, to become functions of $y$ near 1, they must be evaluated at the unique solution of $Y(t)=y$. For completeness, we also write down the function $\rho$.

$$
\begin{aligned}
B_{0}= & \frac{(3 t-1)^{2}(1+t)^{6} \log (1+t)}{512 t^{6}}-\frac{\left(3 t^{4}-16 t^{3}+6 t^{2}-1\right) \log (1+3 t)}{32 t^{3}} \\
& -\frac{(1+3 t)^{2}(1-t)^{6} \log (1+2 t)}{1024 t^{6}}+\frac{1}{4} \log (3+t)-\frac{1}{2} \log (t)-\frac{3}{8} \log (16) \\
& -\frac{\left(217 t^{6}+920 t^{5}+972 t^{4}+1436 t^{3}+205 t^{2}-172 t+6\right)(1-t)^{2}}{2048 t^{4}(1+3 t)(3+t)}, \\
B_{2}= & \frac{(1-t)^{3}(3 t-1)(1+3 t)(1+t)^{3} \log (1+t)}{256 t^{6}} \\
& -\frac{(1-t)^{3}(1+3 t) \log (1+3 t)}{32 t^{3}}+\frac{(1+3 t)^{2}(1-t)^{6} \log (1+2 t)}{512 t^{6}} \\
& +\frac{(1-t)^{4}\left(185 t^{4}+698 t^{3}-217 t^{2}-160 t+6\right)}{1024 t^{4}(1+3 t)(3+t)} \\
B_{4}= & \frac{\log \left(\frac{1+t}{\sqrt{1+2 t}}\right)(1-t)^{6}(1+3 t)^{2}}{512 t^{6}}+\frac{P(1-t)^{5}}{2048 t^{4}(3+t) Q} \\
B_{5}= & -\frac{\sqrt{3} \frac{(1-t)^{6}}{90}\left(\frac{S}{(1+t)^{3 / 2}}\right)^{5 / 2},}{t Q}
\end{aligned}
$$

where

$$
\begin{gathered}
P=-2400+57952 t+303862 t^{2}+466546 t^{3}+264775 t^{4}+76679 t^{5}+11495 t^{6}+739 t^{7} \\
Q=400+1808 t+2527 t^{2}+1155 t^{3}+237 t^{4}+17 t^{5} \\
S=144+592 t+664 t^{2}+135 t^{3}+6 t^{4}-5 t^{5} \\
\rho=\frac{1}{16} \sqrt{1+3 t}(1-t)^{3} t^{-3} \exp (A)
\end{gathered}
$$

where

$$
\begin{aligned}
A= & \frac{\log (1+t)(3 t-1)(1+t)^{3}}{16 t^{3}}+\frac{\log (1+2 t)(1+3 t)(1-t)^{3}}{32 t^{3}} \\
& +\frac{(1-t)\left(185 t^{4}+698 t^{3}-217 t^{2}-160 t+6\right)}{64 t(1+3 t)^{2}(3+t)} .
\end{aligned}
$$


The approximate values of the univariate constants $B_{i}=B_{i}(t)$, where $t=0.62637$ is the unique solution of $Y(t)=1$, are

$$
\begin{array}{ll}
B_{0}=0.73970 \cdot 10^{-3}, & B_{2}=-0.14914 \cdot 10^{-2}, \\
B_{4}=0.76718 \cdot 10^{-3}, & B_{5}=-0.35019 \cdot 10^{-5} .
\end{array}
$$

Finally we include the singular expansion of $U(x, D(x, y))$ at the dominant singularity $R(y)$ :

$$
U(x, D(x, y))=U_{0}(y)+U_{1}(y) X+U_{2}(y) X^{2}+\mathcal{O}\left(X^{3}\right),
$$

where $X=\sqrt{1-x / R(y)}$ and the $U_{i}$ are, as functions of $t$, given by

$$
\begin{aligned}
U_{0} & =\frac{1}{3 t}, \\
U_{1} & =\left(\frac{4(1+3 t)^{2}\left(-5 t^{5}+6 t^{4}+135 t^{3}+664 t^{2}+592 t+144\right)}{27 t^{3}(1+t) Q}\right)^{1 / 2}, \\
U_{2} & =\frac{2(1+3 t) T}{27 t^{2}(1+t)^{2} Q^{2}},
\end{aligned}
$$

where $Q$ is as before and

$$
\begin{aligned}
T= & 691 t^{12}+10112 t^{11}+98693 t^{10}+719346 t^{9}+3723625 t^{8}+13180580 t^{7} \\
& +31133003 t^{6}+47691938 t^{5}+47354348 t^{4}+30156200 t^{3}+11835336 t^{2} \\
& +2596736 t+243072 .
\end{aligned}
$$

\section{ACKNOWLEDGEMENTS}

We are grateful to Philippe Flajolet for his encouragement and useful discussions during our research, to Eric Fusy for his help in deriving large deviation estimates and in simplifying some algebraic expressions, and to Dominic Welsh for giving us access to an early version of [12. Discussions with Manuel Bodirsky and Mihyun Kang are also acknowledged.

\section{REFERENCES}

1. E. A. Bender, Z. Gao, N. C. Wormald, The number of 2-connected labelled planar graphs, Electron. J. Combin. 9 (2002), R43. MR1946145 (2003i:05071)

2. M. Bodirsky, O. Giménez, M. Kang, M. Noy, Asymptotic enumeration and limit laws of series-parallel graphs, Europ. J. Combin. 28 (2007), 2091-2105. MR2351512

3. N. Bonichon, C. Gavoille, N. Hanusse, D. Poulalhon, G. Schaeffer, Planar Graphs, via WellOrderly Maps and Trees, Graphs Combin. 22 (2006), 185-202. MR 2231990 (2007g:05085)

4. A. Denise, M. Vasconcellos, D. J. A. Welsh, The random planar graph, Congr. Numer. 113 (1996), 61-79. MR1393702(97e:05171)

5. P. Flajolet, A. Odlyzko, Singularity analysis of generating functions, SIAM J. Discrete Math. 3 (1990), 216-240. MR.1039294 (90m:05012)

6. P. Flajolet and R. Sedgewick, Analytic combinatorics, to be published in 2008 by Cambridge University Press, preliminary version available at http://algo.inria.fr/flajolet/ Publications.

7. E. Fusy, Quadratic exact-size and linear approximate-size random sampling of planar graphs, Discrete Math. Theor. Comput. Sci. Proc. AD (2005), 125-138. MR2193112

8. S. Gerke, C. McDiarmid, On the Number of Edges in Random Planar Graphs, Combin. Probab. Comput. 13 (2004), 165-183. MR2047234 (2005b:05201)

9. S. Gerke, C. McDiarmid, A. Steger, A. Weissl, Random planar graphs with given average degrees, in Combinatorics, Complexity, and Chance. A Tribute to Dominic Welsh, pp. 83-102, Oxford U. Press, Oxford (2007). MR2314563 (2008b:05161) 
10. O. Giménez, M. Noy, Estimating the growth constant of labelled planar graphs, in Mathematics and Computer Science III: Algorithms, Trees, Combinatorics and Probabilities (M. Drmota, P. Flajolet, D. Gardy, B. Gittenberger, eds.), Birkhäuser, Basel, 2004, pp. 133-139. MR2090501 (2005f:05077)

11. F. Harary, E. Palmer, Graphical Enumeration, Academic Press, New York-London, 1973. MR 0357214 (50:9682)

12. C. McDiarmid, A. Steger, D. Welsh, Random Planar Graphs, J. Combin. Theory Ser. B 93 (2005), 187-205. MR 2117936 (2005k:05221)

13. R. C. Mullin, P. J. Schellenberg, The enumeration of c-nets via quadrangulations, J. Combin. Theory 4 (1968), 259-276. MR0218275 (36:1362)

14. D. Osthus, H. J. Prömel, A. Taraz, On random planar graphs, the number of planar graphs and their triangulations, J. Combin. Theory Ser. B 88 (2003), 119-134. MR.1973264|(2004a:05068)

15. B. A. Trakhtenbrot, Towards a theory of non-repeating contact schemes, Trudi Mat. Inst. Akad. Nauk SSSR 51 (1958), 226-269 (in Russian). MR0107474 (21:6199)

16. G. Turán, On the succinct representation of graphs, Discrete Appl. Math. 8 (1984), 289-294. MR:0749658 (85i:05100)

17. W. T. Tutte, A census of planar triangulations, Canad. J. Math. 14 (1962), 21-38. MR0130841 (24:A695)

18. W. T. Tutte, A census of planar maps, Canad. J. Math. 15 (1963), 249-271. MR0146823 $(26: 4343)$

19. W. T. Tutte, Connectivity in graphs, University of Toronto Press, Toronto, 1966. MR0210617 $(35: 1503)$

20. T. R. S. Walsh, Counting labelled three-connected and homeomorphically irreducible twoconnected graphs, J. Combin. Theory Ser. B 32 (1982), 1-11. MR0649833 (83k:05058a)

21. H. Whitney, Congruent graphs and the connectivity of graphs, Amer. J. Math. 54 (1932), 150-168. MR1506881

Departament de Llenguatges i Sistemes Informàtics, Universitat Politècnica de Catalunya, Jordi Girona 1-3, 08034 Barcelona, Spain

E-mail address: Omer.Gimenez@upc.edu

Departament de Matemàtica Aplicada II, Universitat Politècnica de Catalunya, Jordi Girona 1-3, 08034 Barcelona, Spain

E-mail address: Marc.Noy@upc.edu 